Voix et Images

\title{
Fiction, diction, et autres enchantements narratifs
}

\section{Frances Fortier}

Volume 31, numéro 3 (93), printemps 2006

Élise Turcotte

URI : https://id.erudit.org/iderudit/013244ar

DOI : https://doi.org/10.7202/013244ar

Aller au sommaire du numéro

\section{Éditeur(s)}

Université du Québec à Montréal

\section{ISSN}

0318-9201 (imprimé)

1705-933X (numérique)

Découvrir la revue

Citer cet article

Fortier, F. (2006). Fiction, diction, et autres enchantements narratifs. Voix et Images, 31(3), 134-139. https://doi.org/10.7202/013244ar d'utilisation que vous pouvez consulter en ligne.

https://apropos.erudit.org/fr/usagers/politique-dutilisation/ 


\section{R O M A N}

Fiction, diction, et autres enchantements narratifs

$++$

FRANCES FORTIER

Université du Québec à Rimouski

Picaresque ou minimaliste, arrimée au souvenir individuel ou à l'histoire du monde, retranchée derrière une narration impassible ou traversée de multiples discours, la fiction contemporaine, lorsqu'elle s'énonce à la première personne, sait déjouer les pièges autobiographiques. Aucune fascination narcissique ne nourrit le «je» romanesque, délibérément fictif ou carrément anonyme, à l'origine des trois récits de mémoire rapprochés ici précisément parce qu'ils illustrent divers rapports au réel. Le premier, en toute fantaisie assumée, réinvente le dire biographique; le second, par le biais d'une fabulation métaphorique, transpose la souffrance psychologique; en contrepoint, le troisième envisage la réalité à travers le prisme des atrocités humaines, d'Hiroshima à Bagdad. Réécriture du réel pris en charge par l'imaginaire, ces trois romans présentent en outre des dynamiques formelles inédites, auxquelles il convient de s'attarder.

Vif, échevelé, foisonnant, le dernier roman de Jean-François Chassay, Les taches solaires ${ }^{1}$, fait jouer ensemble, au gré d'épisodes rocambolesques se déroulant sur trois siècles et deux continents, des chiffres et des lettres, un système solaire et des vengeances meurtrières, des dynasties de personnages et des pages d'encyclopédie. «La vie est une stupéfaction, généralement chiffrée» (316) dira le personnage narrateur, ce Charles Bodry de 33 ans né à Montréal en 1974, astrophysicien de son état et qui écrit sous nos yeux, du 19 janvier au 21 décembre 2007 à 11 h 7, en trentetrois chapitres numérotés et chacun portant le nom d'une planète, l'histoire de sa vie et celle de ses ancêtres, du premier Jean Beaudry parisien né en 1732 au dernier Jean Beaudry, mort en 1995 et rebaptisé Bodry sur l'instance de l'avant-dernier, grandpère du narrateur, qui a ainsi décidé de couper court au jeu de massacre œdipien qui faisait s'entretuer tous les Jean Beaudry. Rien n'est laissé au hasard dans ce récit de

$$
++
$$

1 Jean -François Chassay, Les taches solaires, Montréal, Boréal, 2006, 372 p. 
filiation loufoque, qui construit en miroir deux lignées parallèles de Jean Beaudry, celle issue de la première union avec une Josephte de Montréal ignorant tout de l'autre née du mariage du même avec une Louise-Angéline de Louisianne, et dont la révélation sera au cœur d'un enchaînement de «neuf meurtres, comme les neuf planètes» (329), du Jean Beaudry II bis tuant Jean Beaudry $1^{\text {er }}$ pour venger l'honneur familial jusqu'au meurtre de Jean Beaudry V par Jean Beaudry VI bis.

La spécularité affichée de l'anecdote se dédouble encore dans l'autobiographie fictive, montée en parallèle et en alternance alors que chacun des chapitres où le «je» de Charles Bodry relate sa vie présente s'insère entre les plages narratives consacrées aux Beaudry. Jouant de leur double face, mythologique et planétaire, les intitulés des chapitres se voient en outre répétés, un à la suite de l'autre, à l'exception de «Lune» (1 et 33), intitulé qui encadre l'ensemble des récits, et de "Soleil», avec une seule mention (le chapitre 32). Ainsi, à titre d'exemple, le chapitre "28. Vénus» raconte l'apparition de l'amour dans la vie du «je», sous la forme d'une I-BEE (!) de 24 ans qui vient de terminer un mémoire de maîtrise sur une femme astronome, Nicole-Reine Étable de la Brière, née en 1723 ; le chapitre suivant «29. Vénus », met en scène l'histoire d'amour incestueuse entre Jean Beaudry V et sa sœur jumelle Louise-Angéline, journaliste suffragette qui défendra l'égalité des femmes. Et tout comme la chronique familiale multipliait les jeux de miroir et les réduplications, la narration autobiographique va elle aussi doubler les scènes, rapprochant les destins de Charles et de son père, en les affligeant tous les deux du deuil d'une sœur enfant prénommée Jeanne. Cette virtuosité du reflet révèle un imaginaire systémique qui dynamise les boucles narratives à la manière des révolutions planétaires autour du soleil. Haletant, le récit ne révélera qu'à la toute fin le lien qui unit le «je» aux dynasties Beaudry, double lien de fait hérité à la fois de son père et de sa mère, à la faveur de carnets où sont consignés les dessous de cette fable familiale.

Ces genres détournés de leur finalité, le récit de filiation et l'autobiographie, s'écrivent encore sur fonds de roman historique, qui relate dans le détail la déportation des Acadiens, la construction du canal Lachine - pièce maîtresse du discours narratif, qui permet d'unir tous les espaces ici métaphorisés, la terre et le ciel, l'eau et le feu, le bien et le mal, la haine et l'amour -, les conquêtes spatiales, la Révolution française, les luttes féministes, la révolte des Patriotes, l'expédition LewisClark, etc., et où défilent, aux détours des pages, Wojciech Jaruzelski, Giordano Bruno, Anne Frank, Nicolas Berryer, sœur Marie Morin, Pierre-Paul Riquet et tant d'autres visages de la petite et de la grande Histoire. Une telle écriture à contrainte, qui sait lire le monde et dire son foisonnement, s'approprie par le biais d'une narrativité inventive, sans cesse relancée au gré d'un mot, d'une image rabelaisienne, de personnages intratextuels, de bribes de discours doxiques ou savants, les enjeux de l'écriture contemporaine et les met à distance, en toute ironie assumée:

Car il s'agit bien de cela: fabuler, construire un récit en omettant certaines choses parce qu'elles sont trop incompréhensibles, rapprocher des pièces quand elles permettent de faire sens. Parce que qu'est-ce que vivre, sinon une tentative pour trouver les pièces manquantes du puzzle qui nous constitue? Et voilà comment, comme malgré nous, la famille nous fait retour. (107) 
La famille fait aussi retour avec Le mangeur de Ying Chen ${ }^{2}$, mais dans un registre métaphorique qui tient à distance le réel, la rationalité narrative et la représentation. Non située dans le temps ni dans l'espace, cette fable sur l'amour filial joue d'une double isotopie, alimentaire et monstrueuse, la monstruosité héritée d'une arrièregrand-mère faisant du père de la narratrice un «mangeur» compulsif de poissons crus qui finira par avaler sa fille. Cette aïeule, qui, en «l'absence de son mari parti en guerre, dans l'extrême oisiveté, [...] avait élevé un très grand poisson dans un immense aquarium et en avait fait son ami» (10), se retrouve sur le dernier tableau du père peintre, sous une forme pour le moins étrange: "Une minuscule tête de femme sur un gigantesque corps de poisson qui émerge d'un étang bleuâtre. La tête avait entre les dents un morceau de chair difficilement identifiable.» (23) Polysémique, l'image du mangeur tient tout autant de l'ogre que du prédateur sexuel, sans que ces pistes ne soient jamais exploitées, l'univers diégétique ne laissant entrevoir que la singularité d'un monde clos sur lui-même, où n'existent que des dénominations génériques: le père, la mère enfuie, un A. archéologue, «je», l'épicière, un S. et un V. énigmatiques, l'inspecteur, un ami qui doit emmener la narratrice au cinéma à $14 \mathrm{~h}$ 30, figurants d'un drame psychologique dont on devinera l'enjeu, à défaut de bien le saisir.

Une telle actorialisation désincarnée - dans un texte qui n'a de cesse de parler de chair, de viandes, de faim et de dévoration - instaure une tension en réponse à celle de la structure du roman, à l'image de ces tableaux du père, qui «ne font que décrire le saut entre deux points, sans trace et sans ligne au milieu » (96). Le récit du «je», de fait, alterne entre l'évocation de deux espaces, celui du père, et celui de A. son mari, où la porosité du réel et de l'imaginaire vient trouer le déroulement événementiel:

Quant à moi, je ne me demande pas quel désert j'ai traversé sans père, comment et en combien de temps. J'ose à peine me pencher sur ce trou, sur l'intervalle qu'il y a entre deux après-midi ensoleillés de la même manière, sur la distance entre mon père et mon mari A., j'éprouve du vertige. (79)

Ce vertige halluciné repose sur une pareille anticipation, alors que «je» attend d'être avalée par le père ou rêve à son tour de dévorer A. pour en arriver à une «union parfaite, indestructible, absolue» (131). Rien ne sera justifié au plan narratif, ni la disparition du père, ni la rencontre de A., pas plus que le désir du père pour l'épicière bien grasse ou la présence de l'inspecteur de police qui enquête, mais longtemps après le fait, sur la double disparition du père et de la fille, non plus que cet invraisemblable vernissage où la fille, reconnaissant les tableaux du père, décide de revenir sur les lieux de son enfance et de revivre sous nos yeux la scène de

2 Ying Chen, Le mangeur, Montréal, Boréal, 2006, 138 p. 
l'avalement. Ou plutôt tout le sera par cette fin heureuse, où A. ramène le «je» dans son univers, après avoir décrit à l'énigmatique inspecteur son état de santé mentale et expliqué qu'elle renonce à son héritage. L'histoire est classée, en quelque sorte, et le lecteur comprend, avec la narratrice, qu'il fallait

\begin{abstract}
devenir complètement insensible aux souffrances car il ne s'agit jamais que de souffrances, comprendre de plein gré que l'histoire en question n'est rien d'autre qu'un cauchemar, admettre de tout cœur que si je crois qu'un poisson songe à moi, c'est qu'en réalité je songe à un poisson, que tout vient de ma tête, qu'il n'y a là rien de vraiment extraordinaire ou de surnaturel. (35)
\end{abstract}

Ce récit fasciné l'est encore sur un autre plan, dans cet équilibre délicat de l'écriture qui amalgame la saisie réaliste du quotidien à son propre décrochage métaphorique. Porté par une logique du sensible, le récit se déploie à la faveur de petits gestes, de sensations fines qui déportent l'imaginaire: choisir des légumes, dégeler une viande, manger, nettoyer la table, ranger deviennent les événements majeurs d'un récit obnubilé par la couleur rosée de la peau, les démangeaisons, les odeurs, le bain, la digestion, la lumière à la fenêtre, toutes sensations cannibalisées par une narratrice qui se croit «l'arrière-petite-fille d'un poisson» (111).

Tout aussi obnubilé par le souvenir, mais évoluant dans un univers plus concret, le narrateur de Revoir Nevers de Roger Magini ${ }^{3}$, journaliste parisien hanté par la répétition des atrocités que s'inflige une humanité sans mémoire, se réfugie une dizaine de jours au Mexique, le temps d'écrire un article commémorant les soixante ans d'Hiroshima. En narration simultanée, ce «je» décrit le trajet qui le mène de Roissy à Mexico, puis à Puebla et de là à Cholula - lieu de culte précolombien où Hernan Cortès fit massacrer plus de trois mille personnes en 1519 -, réfléchit à l'oubli qui ensevelira demain les tragédies contemporaines du Rwanda, de l'Irak, du Soudan, du Darfour, de l'Afghanistan, visite le site archéologique de Los Remedios, revoit constamment en rêve une femme au kimono fleuri qui a vu Hiroshima: "Six heures ont sonné au carillon de l'église San Pedro, les portières d'un véhicule claquent sous ma fenêtre, je me souviens, Cholula, Hiroshima, ô mes spectres, existez-vous réellement. » (37) Installé chaque jour à la même table d'un café, il fume, lit le journal et tente d'écrire en prêtant une attention distraite à ces Cholultèques occupés à vivre dans un présent immédiat et qui se déplacent au gré de la progression du soleil sur le zócalo: le marchand de gâteaux secs saupoudrés de sucre, les vendeuses de huipils, l'adolescent et ses panamas, la jeune femme et son panier d'arums apparaissent comme autant de silhouettes que le narrateur, tout aussi anonyme, observera à plusieurs reprises et figera à jamais dans une sorte de temps immémorial.

3 Roger Magini, Revoir Nevers, Montréal, Éditions de la Pleine Lune, 2006, 74 p. 
Statique, la narration passe par la description minutieuse de détails, qui traduisent la touffeur ambiante et la torpeur où s'englue le «je», dans cette superposition de lectures - il a lu Abé Kôbô dans l'avion et se sent comme Niki Jumpei le héros de La femme des sables tout en repassant constamment dans sa tête le Hiroshima mon amour de Marguerite Duras - , de souvenirs fugitifs d'une jeune femme à vélo sur les quais de Nevers et de réflexions sur la fragilité humaine. Absorbé dans sa méditation, il reproduit une série de menus gestes qui ne le rapprocheront jamais de quiconque: commander à manger, déposer son guide touristique sur la table, fumer, observer. Cet homme est seul, le texte viendra le réaffirmer à la faveur d'un singulier jeu de focalisation qui fait systématiquement alterner les points de vue, les séquences assumées par le «je» étant toujours encadrées par une narration hétérodiégétique, signalée par des crochets typographiques, qui montre l'homme dans sa solitude, son silence, son malaise:

[Faits et gestes de la vie quotidienne à Cholula. Yeux des femmes assises sur le trottoir; leurs enfants. L'homme arrive devant le marché; les sept allées de l'édifice lui évoquent les sept branches de l'estuaire de la rivière Ota. Il se fraye un chemin parmi la foule; on l'apostrophe, on lui demande ce qu'il désire. Il erre, il ne s'arrête pratiquement jamais. On croirait que rien ne l'intéresse tant son visage est impassible. Il réfléchit à l'article qu'il doit écrire sur Hiroshima; quelques images surgissent, il ne sait pas si elles sont véritables ou imaginaires. Odeurs de fleurs fanées, de chairs putréfiées. Toute précision sur ce qu'il compte écrire est impossible, il l'ignore lui-même.] (23)

$\mathrm{Au}$ bout de quelques jours, l'homme repartira avec son texte écrit, refera le trajet inverse et cette fois feuilletera, dans l'Airbus qui le ramène à Paris, une revue décrivant les charmes de Nevers, «ses relais châteaux pour touristes fortunés, Nevers et son histoire, son passé» (73).

En possession de leur écriture et maîtres de leur art, ces écrivains confirmés font sciemment de leurs «je» fictifs des foyers de discours, de sensations ou de méditations, affirmant ainsi, par le biais d'une narration débridée, impassible ou fermement maintenue, leur appartenance esthétique: Chassay reconduit avec Les taches solaires ce ludisme textuel qui est le sien, friand de contraintes et d'interdiscursivité ; Chen, dans la foulée de ses ouvrages antérieurs, transpose l'écriture du soi dans un roman familial constamment réécrit, alors que Le mangeur propose cette fois une figure de père; Magini, avec Revoir Nevers, précise son ancrage dans une pensée du mémoriel, où le voyage devient partie prenante d'une réappropriation du réel.

Pour distincts qu'ils soient, ces romans proposent les mêmes réponses formelles aux questions de notre temps que sont la mémoire et l'altérité. La surabondance de la figure de répétition - marquée par la reprise systématique de séquences, de motifs, de thèmes, de gestes ou de rêves - , qu'elle vise à inscrire la filiation, la quotidienneté de la vie ou la récurrence des apocalypses, redit haut et 
fort le déterminisme qui nous fait répéter les erreurs et cherche à le tenir en respect, qu'il s'agisse du meurtre des ancêtres, de la transmission génétique ou des drames de l'Histoire. Cette écriture de la mémoire met en outre en relief l'altérité essentielle du sujet, par le va-et-vient constant entre deux univers textuels: les chapitres renvoyant en alternance au présent contemporain du «je» et au passé de ses ancêtres, le rythme régulier qui fait constamment passer le «je» de l'espace de la maison du père à celui du mari ou la délégation systématique de la focalisation, marquée typographiquement, qui montre le «je» tantôt de l'intérieur et tantôt de l'extérieur, sont autant de manières de dire l'écartèlement du sujet, déchiré entre le présent et un passé qui le hante:

J'ai tenté de prendre du recul, de considérer cette situation avec les yeux d'un autre, cela a été une entreprise vaine; le caractère irréversible de l'écoulement du temps me l'a interdit, je suis plongé dans un présent mort auquel je participe malgré moi, mais avec une certaine distance qui m'en sépare, un temps encore dépourvu de mémoire (43-44),

dit le narrateur de Revoir Nevers, en une phrase qui marque bien la singularité de ces jeux de mémoire. 